\title{
Accuracy and precision of the TAS analyser for near-patient INR testing by non-pathology staff in the community
}

\author{
P G Cachia, E McGregor, S Adlakha, P Davey, B M Goudie
}

\begin{abstract}
Aims-To assess the accuracy and precision of INR measurement by trained practice and district nursing staff using the Thrombolytic Assessment System (TAS) analyser.

Methods-Seventeen nurses from four practices were trained to measure INR using the TAS analyser on citrated capillary blood samples. Quality control (QC) consisted of: daily internal QC using normal and abnormal commercial plasmas; monthly local external QC scheme using fresh citrated venous blood; and registration of all analysers in the NEQAS (national external quality assessment scheme) main users scheme.

Results-Analysis of internal QC results demonstrated satisfactory interanalyser and intra-analyser precision with no evidence of analytical drift in any of the four practice analysers over an eight month period. Local and national external QC results confirmed the interanalyser precision but INR was underestimated by the TAS analysers compared with the CA 1000 using either Diagen rabbit brain thromboplastin or Innovin, and with other NEQAS users.
\end{abstract}

Conclusions-The TAS analyser has many features to commend it for use by nonpathology staff to determine INR. Local internal and external QC and entry into the NEQAS main users group are possible because the TAS analyses citrated plasma or blood. The TAS analyser underestimates INR when the geometric mean normal prothrombin time (GMNPT) is determined by conventional methods. A local correction factor can be introduced by adjusting the normal PT to give INR results comparable with the local laboratory. This is particularly desirable when INRs are measured using both nearpatient and laboratory analytical systems on different occasions.

(f Clin Pathol 1998;51:68-72)

Medicines Monitorin Unit, University of Dundee, Dundee, UK P Davey

Westgate Health Centre, Dundee, UK B M Goudie

Correspondence to: Dr Cachia.

email: p.g.cachia

(a)dundee.ac.uk

Accepted for publication 11 November 1997 ering anticoagulant services include hospital, surgery, pharmacy or other community settings by various professional staff (including doc- tors, nurses, pharmacists, and medical laboratory scientific officers (MLSOs)). The attraction of a near-patient anticoagulant monitoring service is that INR testing and advice on anticoagulant dosing can be provided in a single consultation. A community based service has the added advantage that more staff time may be available for explanation and patient education than in a traditional hospital anticoagulant clinic. For a community based system to improve patient care it is essential that the INR results produced by non-pathology staff using portable coagulometers are accurate and precise. Expert groups recently have suggested quality standards for near-patient testing by non-pathology staff ${ }^{3}$ and for INR testing using portable coagulometers. ${ }^{4}$ The quality standards of the Joint Working Party on Quality Assurance have subsequently been adopted by Clinical Pathology Accreditation (CPA) Ltd. ${ }^{5}$ One published study compared INR results obtained by laboratory MLSO staff using the Biotrack 512 (Ciba Corning, Halstead, Essex, UK) with laboratory analysis of paired patient samples over a short period of time. ${ }^{6}$ There is, however, no published data assessing the accuracy and precision of portable coagulometers operated by trained, non-pathology staff during a prolonged period of patient monitoring in the community.

We report such a study in which patients attending four general practices were monitored by practice or district nurses who had been trained to use the Thrombolytic Assessment System (TAS) analyser (Diagnostic Testing Ltd, Bury St Edmunds, Suffolk, UK) to determine INR and then offer immediate warfarin dosing advice.

\section{Materials and methods}

ANALYSER

The TAS analyser was selected because of the features shown in table 1 . The TAS analyser also has a user friendly LCD panel and keypad for entering data, detachable AC power supply, internal battery, and general robust construction. Citrated plasma or whole blood is added to the reaction chamber in a single use prothrombin time (PT-ONE) test card. The reaction chamber contains paramagnetic particles that move in an electromagnetic field that is turned on and off during operation. Cessation of movement of the paramagnetic particles when a blood clot forms is detected by a photodetector.

TRAINING SCHEME

Seventeen practice or district nurses underwent three, one hour training sessions at the 
Table 1 Advantages of the TAS analyser for near-patient testing service involving multiple testing sites and designated staff

\begin{tabular}{ll}
\hline Feature & Advantage \\
\hline Operator ID number & Multiple operators in four practices \\
Supervisor mode & Operation by non-pathology staff \\
Analyser memory & Record of patient data, contol data, operator \\
User programmable normal PT & Compliance with standard practice \\
Use of control plasma for internal QC & Independent measure of precision and drift \\
Participation in NEQAS and EQAS & Comparison with other analytical systems \\
\hline
\end{tabular}

haematology department where haematology medical and MLSO staff provided instruction on obtaining citrated capillary blood, operating the TAS analyser, performing quality control, advising on warfarin dose adjustment, and criteria for referring patients for medical advice. A final two hour visit to the hospital anticoagulant clinic enabled nursing staff to perform INR testing and provide advice on dosing to patients. On completion of the training scheme a certificate of competence was issued and each nurse given an identification number for operating that practice's TAS analyser. Standard operating procedures, drawn up by haematology staff, were included with each analyser's logbook.

NORMAL PROTHROMBIN TIME DETERMINATION Citrated capillary blood was obtained using a capillary sampling device (Kabe Laboratories, Numbrecht-Elsenroth, Germany) in which blood was collected in a $150 \mu \mathrm{l}$ capillary tube and tipped into a plastic vial containing $16.7 \mu \mathrm{l}$ of $0.1 \mathrm{M}$ citrate. The geometric mean normal prothrombin time (GMNPT) was determined according to established criteria. ${ }^{7}$ Paired venous (Vacutainer, nine parts blood to one part $0.105 \mathrm{M}$ citrate) and citrated capillary samples were obtained from 20 normal blood donors and the prothrombin times (PTs) determined on each of five TAS analysers. There was no significant difference between the five analysers' mean capillary or venous PTs (data not shown). However, there was a significant difference between the mean capillary and mean venous PTs for individual analysers and for the pooled results from all five analysers (11.89 v 11.37 seconds, $\mathrm{p}<0.02$, paired $t$ test). The analysers were therefore programmed to use a normal PT of 11.9 seconds for testing capillary samples and 11.4 seconds for testing venous blood samples. The normal PT differed by up to 2.1 seconds (11.1 to 13.2 , manufacturer's data) for different batches of PT cards. It was therefore necessary to determine the normal PT each time a new batch of cards was introduced (four times during the course of the study).

QUALITY CONTROL

Internal quality control

Laboratory MLSO staff determined the mean (3 SDs) PTs from 10 replicate tests for each batch of normal and abnormal control plasmas on the laboratory TAS. The control plasma was then divided into $50 \mu \mathrm{l}$ aliquots in $500 \mu \mathrm{l}$ capped polypropylene tubes, frozen, and kept in the freezer compartment of a domestic freezer in each of the practices. Internal QC with both normal and abnormal control plasmas was performed by one of the designated nursing staff each day that their analyser was used for INR determination. The results were entered into the analysers' logbooks. Analysis of individual practice internal QC results was performed on data downloaded from the TAS memory to a computerised spreadsheet program (Microsoft Excel). Nursing staff were required to contact haematology MLSO staff if duplicate control samples from either normal or abnormal controls were both outside the stated ranges. Patient testing would not proceed until MLSO staff had excluded any faults in the analytical system.

\section{External quality control}

An external quality assessment scheme (EQAS) was run from the haematology laboratory. From September 1995 to May 1996, 20 fresh, citrated venous whole blood samples from patients receiving warfarin were sent to the practices for analysis on the four TAS analysers by practice staff and the results compared with the laboratory TAS analyser and standard analytical system (Sysmex CA 1000 initially using Diagen rabbit brain thromboplastin (Diagnostic Reagents, Thame, Oxfordshire, UK) but changing to Innovin (Dade, Miami, Florida, USA) from March 1996). All four practices and the laboratory TAS analysers were entered into the national external quality assessment scheme (NEQAS) main users group for surveys 94-98.

\section{Results}

INTERNAL QUALITY CONTROL

Precision

The mean PTs and coefficients of variation (CVs) for each of the four practice TAS analysers are shown in table 2 . There were no significant differences ( $t$ test) in the mean PTs for either normal or abnormal controls. The CVs ranged from $6.72-7.73 \%$ for the normal control plasmas and from $7.61-13.4 \%$ for the abnormal control plasmas. On 10 occasions MLSO staff were required to visit practices because control plasma results were outside the designated ranges on duplicate testing. The fresh control sample brought from the laboratory was always within the designated range during the investigation of these problems suggesting that samples may deteriorate during storage in a domestic freezer.

\section{Analytical drift}

There was no evidence of analytical drift in any of the four surgery based analysers during the study (fig 1).

Table 2 Internal QC from four TAS analysers with both normal and abnormal control plasmas

\begin{tabular}{lcccc}
\hline & TAS 1 & TAS 2 & TAS 3 & TAS 4 \\
\hline Normal control & & & & \\
Number of tests & 181 & 166 & 152 & 160 \\
Mean time (s) & 12.8 & 11.9 & 11.9 & 12.5 \\
CV (\%) & 7.41 & 7.07 & 6.72 & 7.73 \\
Abnormal control & & & & \\
Number of tests & 179 & 168 & 160 & 152 \\
Mean time (s) & 28.5 & 27.0 & 28.0 & 28.8 \\
CV (\%) & 13.4 & 10.2 & 7.61 & 8.31 \\
\hline
\end{tabular}



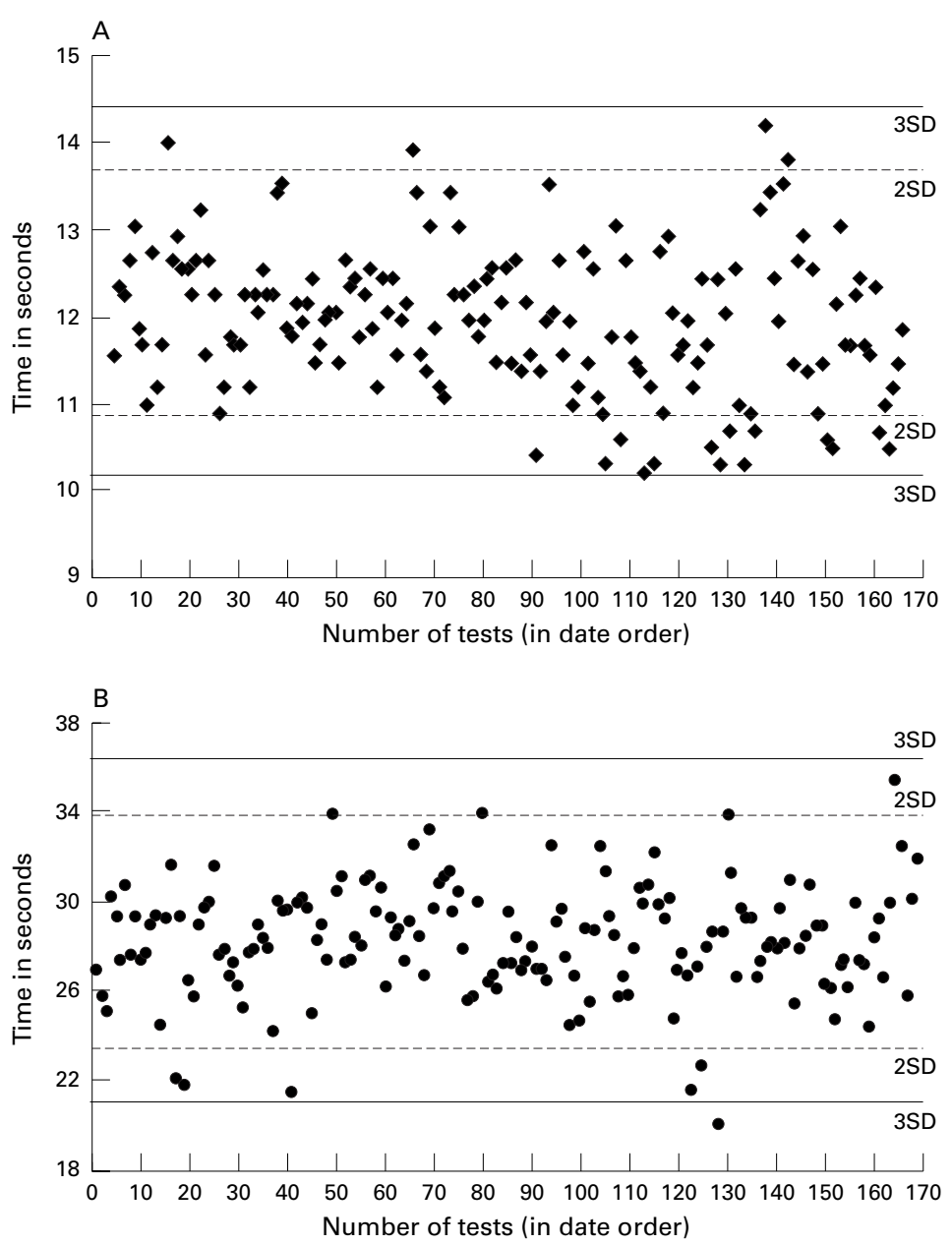

Figure 1 Prothrombin time (PT) results from practice 2 using the TAS analyser on $(A)$ normal and (B) abnormal control plasmas measured over an eight month period. Normal control plasmas: mean PT, 12.3 seconds; (2 SD) 10.9-13.7 seconds; (3 SD) 10.2-14.4 seconds. Abnormal control plasmas: mean PT, 28.7 seconds; (2 SD) 23.6-33.8 seconds; (3 SD) $21.0-36.4$ seconds.

LOCAL EXTERNAL QUALITY CONTROL SCHEME Precision

The CVs for all five TAS analysers for each of the 20 EQAS exercises ranged from $3.2-19.2 \%$ (mean $7.6 \%$ ). The $\mathrm{CV}$ was greater than $10 \%$ in only three of 20 exercises: sample 4 (mean $2.01, \mathrm{CV} 12.9 \%$ ) and sample 9 (mean 3.14 , CV $11.3 \%$ ) (table $3 \mathrm{~A}$ ), and sample 11 (mean 2.53, CV 19.2\%) (table 3B). There was no significant difference ( $t$ test) between the mean INR of all 20 EQAS exercises between the five analysers (table $3 \mathrm{~A}$ and $\mathrm{B}$ ).

\section{Accuracy}

The first 10 samples were tested when the laboratory analytical system used Diagen rabbit brain thromboplastin (table 3A). For each exercise, the mean TAS INRs were less than the CA 1000 and the overall mean TAS INR for all exercises (2.35) was $17.8 \%$ less than the mean CA 1000 INR (2.86), ( $\mathrm{p}<0.001, t$ test). The CA 1000/Diagen RBT performance in NEQAS was satisfactory during this period with all seven exercises within consensus. The later 10 EQAS exercises were performed after the laboratory analytical system had changed to using Innovin (table $3 \mathrm{~B}$ ). The difference between the CA 1000/Innovin and TAS INRs was more pronounced with the overall mean TAS INR (2.2) being $41.1 \%$ lower than the CA 1000/Innovin (3.72), ( $\mathrm{p}<0.0001, t$ test). However, this was partly because of an overestimate of the INR by the CA 1000/Innovin as shown by five of eight NEQAS exercise results being outside (above) consensus (data not shown). Following local International Sensitivity Index (ISI) calibration the CA 1000/Innovin results were within consensus for the next eight exercises (surveys 100-103). The mean TAS INRs (from a single analyser) for these same exercises were $18.5 \%$ lower than the CA 1000/Innovin INRs. Thus the TAS analyser underestimated the INR compared with either the CA 1000/Diagen RBT or the CA 1000/Innovin when both laboratory systems were within NEQAS consensus.

\section{NATIONAL EXTERNAL QUALITY ASSURANCE} SCHEME

Precision

The CVs of the mean INRs from the five TAS analysers ranged from $2.1-13.9 \%$ (mean $7.6 \%$ ) for the 11 samples in NEQAS exercises 94-98. The CV was > $10 \%$ for three of the 11 samples: 95/30 (mean 2.78, CV 10.8\%), 95/41 (mean 3.75, CV 11.3\%), and 95/42 (mean 2.82 , CV $13.8 \%$ ) (table 4). There was no significant difference ( $t$ test) between the mean INRs of all 11 NEQAS exercises between the five analysers (table 4).

\section{Accuracy}

Table 4 shows the INR results of all 11 exercises along with the NEQAS median INRs. The overall mean of the TAS analysers for all exercises was 2.44 compared with an overall NEQAS median of $2.80 \quad(12.9 \%$ below NEQAS median). Twenty two of $55(43.6 \%)$ of the results from all TAS analysers were within consensus.

\section{LOCAL CORRECTION OF NORMAL PROTHROMBIN} TIME

Venous blood samples from 20 patients receiving warfarin (INR range 1.3-6.9, CA 1000) were selected and the PTs for each of these samples determined on the laboratory TAS analyser. TAS INR results were calculated for a series of different normal PTs (range 9.4-11.9 seconds). The regression line derived from a normal PT of 9.6 seconds most closely approximated with the CA 1000 results for this batch of PT cards (fig 2). The five TAS analysers were reprogrammed to a normal PT of 9.6 seconds and the effect of the correction assessed by performing an additional EQAS exercise with four fresh venous samples (table 3C, samples 1-4). The mean TAS and CA 1000 INRs were 2.88 and 3.03, respectively. The results of four subsequent EQAS exercises covering the therapeutic range over a four month period are shown in table 3C (samples 5-8). For these eight EQAS exercises performed after the introduction of local ISI calibration of the CA 1000/Innovin and using the local correction of TAS normal PTs, the difference between the mean INRs of all five TAS 
Table 3 Local EQAS scheme results from five TAS analysers compared with laboratory system

\begin{tabular}{|c|c|c|c|c|c|c|}
\hline Sample & TAS 1 & TAS 2 & TAS 3 & TAS 4 & $\begin{array}{l}\text { TAS } 5 \\
(l a b)\end{array}$ & $\begin{array}{l}\text { CA1000 } \\
\text { (Diagen) }\end{array}$ \\
\hline \multicolumn{7}{|c|}{ A-Compared with CA 1000 using Diagen rabbit brain thromboplastin } \\
\hline 1 & 1.43 & 1.56 & 1.43 & 1.43 & 1.43 & 2.10 \\
\hline 2 & 2.10 & 1.98 & 1.87 & 2.40 & 2.15 & 3.20 \\
\hline 3 & 2.82 & 2.52 & 2.92 & 2.52 & 2.52 & 4.27 \\
\hline 4 & 1.67 & 2.00 & 2.40 & 2.00 & 2.00 & 1.72 \\
\hline 5 & 1.87 & 1.78 & 1.67 & 2.10 & 1.93 & 2.10 \\
\hline 6 & 2.50 & 2.40 & 2.82 & 2.40 & 2.50 & 3.00 \\
\hline 7 & 4.26 & 4.11 & 4.26 & 3.96 & 3.89 & 4.56 \\
\hline 8 & 2.19 & 2.50 & 2.26 & 2.66 & 2.50 & 2.44 \\
\hline 9 & 3.06 & 3.56 & 3.46 & 2.77 & 2.87 & 3.27 \\
\hline 10 & 1.14 & 1.22 & 1.14 & 1.34 & 1.22 & 1.89 \\
\hline Mean & 2.30 & 2.36 & 2.42 & 2.36 & 2.30 & 2.86 \\
\hline \multicolumn{7}{|c|}{ B-Compared with CA 1000 using Innovin (before local ISI calibration) } \\
\hline 11 & 2.26 & 3.36 & 2.50 & 2.36 & 2.19 & 4.48 \\
\hline 12 & 2.19 & 1.90 & 2.05 & 2.05 & 1.90 & 2.79 \\
\hline 13 & 2.36 & 2.26 & 2.26 & 2.26 & 2.55 & 4.16 \\
\hline 14 & 3.6 & 3.41 & 3.13 & 3.32 & 3.32 & 6.37 \\
\hline 15 & 2.00 & 2.20 & 1.90 & 2.00 & 2.00 & 3.60 \\
\hline 16 & 1.20 & 1.20 & 1.30 & 1.40 & 1.40 & 1.50 \\
\hline 17 & 2.26 & 2.07 & 1.88 & 1.88 & 2.07 & 2.56 \\
\hline 18 & 2.55 & 2.26 & 2.17 & 2.17 & 2.36 & 4.90 \\
\hline 19 & 1.89 & 1.79 & 1.89 & 1.79 & 1.89 & 2.35 \\
\hline 20 & 2.18 & 1.99 & 2.18 & 2.18 & 2.18 & 4.44 \\
\hline Mean & 2.25 & 2.24 & 2.13 & 2.14 & 2.19 & 3.72 \\
\hline \multicolumn{7}{|c|}{$\begin{array}{l}\text { C-Compared with CA } 1000 \text { using Innovin (after local ISI calibration) after introduction of locally } \\
\text { corrected normal prothrombin time }\end{array}$} \\
\hline 1 & 1.99 & 1.99 & 1.99 & 2.13 & 2.13 & 1.68 \\
\hline 2 & 3.16 & 3.07 & 2.77 & 3.10 & 3.16 & 3.17 \\
\hline 3 & 3.26 & 3.16 & 2.97 & 2.96 & 2.87 & 3.22 \\
\hline 4 & 3.55 & 3.55 & 3.16 & 3.61 & 3.75 & 4.05 \\
\hline 5 & 2.58 & 2.48 & 2.18 & 1.99 & 2.18 & 2.15 \\
\hline 6 & 3.07 & 3.07 & 2.77 & 3.07 & 2.97 & 3.94 \\
\hline 7 & 4.14 & 3.90 & 3.55 & 3.85 & 3.94 & 4.27 \\
\hline 8 & 3.16 & 3.40 & 3.07 & 3.07 & 2.87 & 2.73 \\
\hline Mean & 3.11 & 3.08 & 2.81 & 2.97 & 2.98 & 3.15 \\
\hline
\end{tabular}

Table 4 Results of NEQAS exercises 94-98

\begin{tabular}{lllllll}
\hline & TAS 1 & TAS 2 & TAS 3 & TAS 4 & $\begin{array}{l}\text { TAS } 5 \\
\text { (lab) }\end{array}$ & $\begin{array}{l}\text { NEQAS } \\
\text { median }\end{array}$ \\
\hline $95 / 30$ & 2.62 & 2.75 & 2.82 & 2.45 & 3.24 & 2.70 \\
$95 / 31$ & 2.62 & 2.82 & 2.62 & 2.40 & 2.50 & 3.00 \\
$95 / 32$ & 1.44 & 1.36 & 1.5 & 1.46 & 1.66 & 1.82 \\
$95 / 41$ & 3.45 & 3.58 & 4.47 & 3.77 & 3.47 & 4.00 \\
$95 / 42$ & 2.82 & 2.57 & 3.46 & 2.82 & 2.45 & 2.84 \\
$96 / 01$ & 2.8 & 3.35 & 3.26 & 3.01 & 3.26 & 3.97 \\
$96 / 02$ & 1.15 & 1.14 & 1.14 & 1.15 & 1.05 & 1.38 \\
$96 / 12$ & 1.94 & 1.62 & 1.83 & 1.94 & 2.04 & 2.20 \\
$96 / 13$ & 2.46 & 2.68 & 2.57 & 2.57 & 2.89 & 2.93 \\
$96 / 15$ & 2.25 & 2.15 & 2.15 & 2.15 & 2.15 & 2.56 \\
$96 / 16$ & 2.46 & 2.57 & 2.25 & 2.36 & 2.57 & 3.34 \\
Mean & 2.37 & 2.42 & 2.55 & 2.37 & 2.48 & 2.80 \\
\hline
\end{tabular}

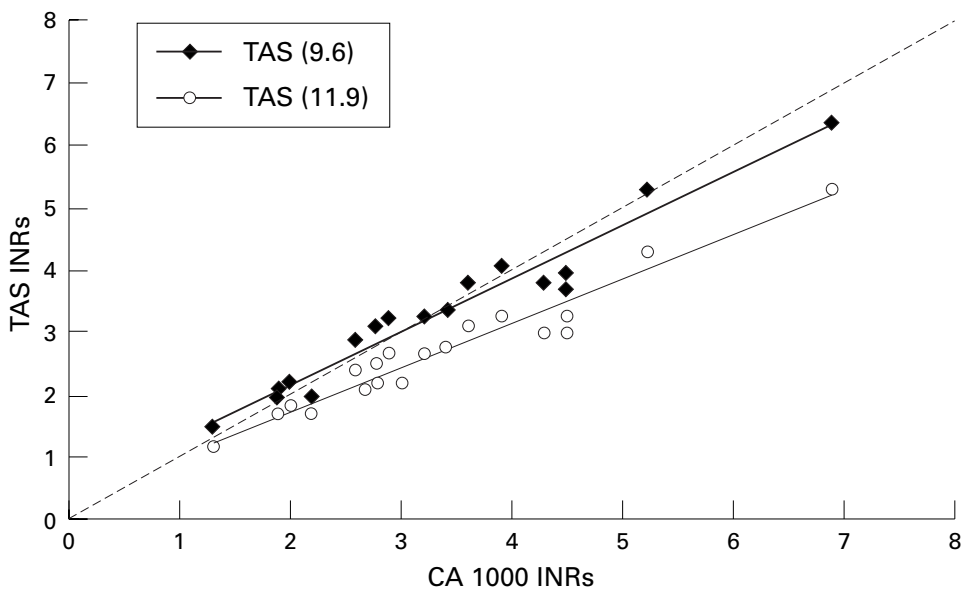

Figure 2 INR results from 20 patients receiving warfarin (range 1.3-6.9, CA 1000) against the laboratory TAS results using the GMNPT calculated from normal plasmas (11.9 seconds) and a normal PT of 9.6 as the closest match to the CA 1000 INR results. analysers (2.99) and the mean CA 1000/ Innovin (3.15) was $5.1 \%$ (not significant, $t$ test).

\section{Discussion}

This study aimed at implementing a nearpatient anticoagulant service complying with all the quality standards defined by the BSH Near Patient Testing Working Party. Analysis of the QC results confirms that the TAS analyser has many attributes to commend it for use in decentralised INR testing by trained, non-pathology staff. The availability of the operator ID number, supervisor mode, and the analyser's memory greatly facilitate essential documentation and permit a detailed audit trail to be performed.

For both internal and external QC the TAS has a major advantage over other near-patient testing instruments that analyse uncitrated whole blood. The range of citrated plasma or whole blood reagents that can be used for QC for the TAS are the same as for any laboratory analyser and entry into the main users group in NEQAS is possible. This is desirable as the accuracy of the TAS INR compared with all other analytical systems can be determined. The NEQAS near-patient testing users group uses uncitrated freeze dried plasma as a control material; therefore, it can be used only to assess the precision between different capillary blood testing systems but not their accuracy against all NEQAS users. Participation in the main users group and the local EQAS were necessary to detect the consistent underestimate of INR by the TAS analysers when the standard practice of determining GMNPT was followed. ${ }^{7}$

The internal QC results demonstrate good reproducibility with no analytical drift in the course of eight months. Commercial control plasmas were inexpensive (less than $£ 1$ for consumables for each test) but laboratory MLSO time was required to aliquot the control plasma in single use tubes and deliver racks of frozen aliquots to the surgery. In addition, the few occasions when duplicate control samples were both out of range probably resulted from sample deterioration during storage in a domestic freezer in the practice. This may have contributed substantially to the variation in control PTs and thus the CVs shown in table 2, which may therefore underestimate the analytical precision that could be achieved by the TAS with a stable control. The problems of storage of control material could be solved by using freeze dried control preparations provided by the manufacturers although this would substantially increase the cost of QC and limit the material available for QC.

The local EQAS using fresh citrated whole blood has the advantage that the control material is the closest possible match to the samples used for INR measurement in patients. However, the scheme required MLSO time to select patient samples, determine the INR on the laboratory TAS and main analysers, and distribute the samples to the participating practices. A larger scheme with multiple community sites for near-patient testing would 
require dedicated MLSO time and could present problems in distributing the fresh venous control samples to geographically distant sites. The differences in INR results between the TAS and Diagen RBT (17.8\%) and subsequently between the TAS and Innovin $(18.5 \%)$ were greater than thromboplastin specific differences previously reported ${ }^{8}$ and would have a significant impact on warfarin dose and level of anticoagulation. It is highly desirable that two different analytical systems, which may be used for the same patient population at different times (for example, home monitoring and hospital/clinic attendance) provide INR results that match as closely as possible. It is not possible to perform a local ISI calibration ${ }^{9}{ }^{10}$ for the TAS because the ISI contained in the magnetic strip for each batch of PT cards cannot be altered. Adjusting the normal PT to give INR results that match the local hospital laboratory is possible with the TAS because of the user programmable normal PT. This approach does not follow the accepted practice of determining a local GMNPT from normal plasmas. ${ }^{7}$ However, it is now established that different INR analytical systems do produce different INR results in spite of the correction of thromboplastin related differences by the incorporation of the ISI. ${ }^{8}$ Local calibration is an attractive option for correcting for differences in INR analytical systems. ${ }^{910}$ We have shown that near-patient testing analysers may perform differently from laboratory analytical systems when standard laboratory practice is followed. The TAS analyser can be recommended for community based near-patient INR testing both because of the flexibility of QC options and the possibility of adjusting the normal PT so that near-patient testing and the local laboratory INRs are closely matched. In view of the rapidly increasing use of near-patient INR analysers in a variety of clinical settings there is an urgent need for a standardised approach to analytical QC and local INR determination.

This work was supported by the Scottish Office Primary Care Development Fund and the Tayside Health Board. The NEQAS survey medians reproduced by kind permission of NEQAS. We are grateful to the medical and nursing staff who participated in this project and to Professor M J Pippard for his helpful comments.

1 Gage BF, Cardinalli AB, Albers GW, et al. Cost-effectiveness of warfarin and aspirin for prophylaxis of stroke in patients with nonvalvular atrial fibrillation. $\mathcal{F A M A}$ 1995;274:183945.

2 Lip GYH, Lowe GDO. Antithrombotic treatment for atrial fibrillation. BMF 1996;213:45-9.

3 Joint Working Party on Quality Assurance. Appendix: Guidelines on the control of near-patient tests and procedures performed on patients by non-pathology staff. In: Wood E, ed. Standard haematology practice 2, Oxford: Blackwell Science, 1994:278-9.

4 England JM, Hyde K, Lewis SM, et al. Guide-lines for near patient testing: haematology. Clin Lab Haem 1995;17:30110 .

5 Clinical Pathology Accreditation (UK) Ltd. Accreditation handbook. Version 9.2. 1995:Appendix 3.

6 Jennings I, Luddington RJ, Baglin T. Evaluation of the Ciba Corning Biotrack 512 coagulation monitor for the contro of oral anticoagulation. F Clin Pathol 1991;44:950-3.

7 British Committee for Standards in Haematology. Guidelines on oral anticoagulation: second edition $f$ Clin Pathol 1990;43:177-83.

8 Kitchen S, Walker ID, Woods TA, et al. Thromboplastin related differences in the determination of international normalised ratio: a cause for concern? Steering Committee of the UK National Quality Assessment Scheme in Blood Coagulation. Thromb Haemost 1994;72:426-9.

9 Pi DW, Raboud JM, Filby C, et al. Effect of thromboplastin and coagulometer interaction on the precision of the International Normalised Ratio. F Clin Pathol 1995;48:13-17.

10 Poller L, Triplett DA, Hirsh J, et al. The value of plasma calibrants in correcting coagulometer effects on international normalized ratios. Am f Clin Pathol 1995;103:358- 